\title{
Multifocal Micronodular Pneumocyte Hyperplasia and Lymphangioleiomyomatosis in Tuberous Sclerosis with a TSC2 Gene
}

Hiroshi Maruyama, M.D., Kuniaki Seyama, M.D., Junko Sobajima, M.D., Kazumichi Kitamura, M.D., Tooru Sobajima, M.D., Tomokazu Fukuda, Ph.D., Kaoru Hamada, M.D., Masahiro Tsutsumi, M.D., Okio Hino, M.D., Yoichi Konishi, M.D.

Departments of Pathology (HM), Internal Medicine (JS, KK), and Thoracic Surgery (TS), Hoshigaoka Koseinenkin Hospital, Hirakata, Japan; Department of Respiratory Medicine (KS), School of Medicine, Juntendo University, Tokyo, Japan; Department of Experimental Pathology (TF, OH), Cancer Institute, Tokyo, Japan; and Second Department of Internal Medicine (KH) and Department of Oncological Pathology (MT, KY), Cancer Center, Nara Medical University, Kashihara, Japan

A 45-year-old woman with a long-standing diagnosis of tuberous sclerosis (TSC) is presented. She has multifocal micronodular pneumocyte hyperplasia (MMPH) and lymphangioleiomyomatosis (LAM) of the lung, together with the detection of TSC2 gene mutation. During surgery for spontaneous pneumothorax, an open-lung biopsy was performed. Micronodules were well defined, measuring approximately $4 \mathrm{~mm}$ in diameter. These MMPHs were histologically composed of papillary proliferation of Type II pneumocytes, with positive immunoreactivity of keratin and surfactant apoprotein. The cystlike spaces, with dilatation and destruction of air spaces, were diffusely formed, and the walls were composed of the spindle cells. Such LAM showed positive immunoreactivity for HMB-45 (a monoclonal antibody specific for human melanoma) and tuberin (the gene product of TSC2). On germline mutation analysis using leukocytes of the present patient, a TSC2 gene mutation was confirmed as a deletion of $G$ (or $g$ ) on Exon 9 by polymerase chain reaction-single-strand conformational polymorphism. However, no mutation was detected in her son. With microdissection analysis using paraffinembedding lung tissues, LOH of the TSC2 gene preliminarily was detected in a LAM lesion but not in MMPH. It is suggested that MMPH, in addition to LAM, could be another pulmonary lesion in TSC

Copyright (C) 2001 by The United States and Canadian Academy of Pathology, Inc.

VOL. 14, NO. 6, P. 609, 2001 Printed in the U.S.A.

Date of acceptance: January 30, 2001.

Address reprint requests to: Hiroshi Maruyama, M.D., Department of Pathology, Hoshigaoka Koseinenkin Hospital, 8-1, 4-chome, Hoshigaoka, Hirakata, Osaka 573-8511, Japan; e-mail: hiromaruyama@sannet.ne.jp; fax: 81-72-840-2266. patients and that the detection of TSC2 and/or TSC1 gene could essentially be useful for the pathogenesis of MMPH and LAM in TSC patients.

KEY WORDS: Loss of heterozygosity (LOH), Lymphangioleiomyomatosis (LAM), Multifocal micronodular pneumocyte hyperplasia (MMPH), PCRSSCP, TSC2 gene, Tuberin, Tuberous sclerosis (TSC).

Mod Pathol 2001;14(6):609-614

Tuberous sclerosis (TSC) is an autosomal-dominant disorder with a relatively high penetrance (90-95\%) and an incidence of $0.58 \sim 1.0$ per 10,000 births (1). Known as the Bourneville-Pringle syndrome, it was described on the basis of hamartomatous lesions in the cerebral cortex and categorized as a phacomatosis including Von Recklinghausen disease and Von Hippel-Lindau disease. TSC is clinically characterized by classic Vogt's triad, including adenoma sebaceum of skin, mental retardation, and epilepsy, but is currently diagnosed by using the criteria of Gomez (2). The criteria were composed of three major categories: primary features such as subependymal nodes, astrocytic retinal hamartomas, fibrous forehead plaques and facial angiofibroma; secondary features such as renal angiomyolipoma and pulmonary lymphangioleiomyomatosis (LAM); and tertiary features such as hypomelanotic macules. Those diagnostic criteria provide a definite clinical diagnosis of TSC under the basis of hamartomatous lesions in organs, chiefly including the brain, retina, skin, heart, kidney, and lung, although incomplete symptom types of TSC have been pointed out. Recently, two predisposing genes have been found in TSC, and it has been reported that there are almost equal percentages of genetic link- 
age to TSC1 at 9q34 and TSC2 at16p13.3 and that up to $60 \%$ of cases are sporadic (3).

It has been reported by Popper et al. (4) that multifocal micronodular pneumocyte hyperplasia (MMPH) in TSC patients might be a new and representative pulmonary lesion, in addition to LAM. Although Kimura et al. (5) reported that the tissue expressions of tuberin in the hamartomas of lung were negative, there are few data on the LAM and $\mathrm{MMPH}$ in TSC.

In the present paper, we report a case of a 45year-old woman who demonstrated both $\mathrm{MMPH}$ and LAM under a long-standing diagnosis of TSC, and we confirmed the detection of a germline mutation of the TSC2 gene and loss of heterozygosity (LOH) in LAM.

\section{CASE REPORT}

A 32-year-old woman (who had one son) was admitted to the Department of Thoracic Surgery via Internal Medicine in Hoshigaoka Koseinenkin Hospital for chest pain due to left-sided pneumothorax. She had been diagnosed as TSC because of facial angiofibroma without obvious epilepsy or mental retardation when she was 13 years old. Moreover, abdominal ultrasound echograms and computedtomography scans had radiographically demonstrated multiple angiomyolipomatous lesions in both kidneys and liver. Chest radiograph on admission showed a left-sided pneumothorax, and intercostal suction drainage was performed. Because of the air leakage, a surgical operation (left axillar thoracotomy) and open-lung biopsies (two specimens, about $3 \times 2 \times 2 \mathrm{~cm}$, from the apex of left upper lobe $\left(S^{1+2}\right)$ and left lower lobe $\left.\left(S^{6}\right)\right)$ were conducted, appearing as a gross bulla formation and multiple small, cystlike spaces of approximately $5 \mathrm{~mm}$ in size. Simultaneously, skin biopsies were taken from face and foot. Serum $\alpha$-fetoprotein and carcinoembryonic antigen (CEA) values were within the normal range at 4 and $1.3 \mathrm{ng} / \mathrm{mL}$, respectively. She was discharged with left lung fully expanded 2 weeks after surgery, and sometimes consulted our hospital. Twelve years thereafter, the patient (at 44 years of age) reconsulted our hospital because of left anterior chest and back pain. A chest radiograph showed diffusely radiolucent and slight reticular shadows, similar to those seen formerly. A chest computed-tomography scan demonstrated marked dilatation and destruction of air spaces and also showed cyst-like spaces. Lung function test yielded an FEV1.0 of $1.94 \mathrm{~L}$ (67.1\%), \%VC of $109.9 \%$, a flow of $\mathrm{V} 25 / \mathrm{HT}$ of $0.32 \mathrm{l} / \mathrm{s} / \mathrm{m}$, and \%DLco of $58.6 \%$, corresponding to obstructive disturbance. For germline mutation analysis, blood samples from the patient and her son were taken. She is now 45 years old and living a normal daily life with slight respiratory functional disturbance, 13 years after the surgery for pneumothorax.

\section{MATERIALS AND METHODS}

The lung, skin and other tissues were fixed in $20 \%$ neutral formaldehyde solution, embedded in paraffin after routine processing, sectioned and stained with hematoxylin and eosin (H\&E), elastica van Gieson (EVG), silver impregnation, and periodic acid-Schiff solutions. Immunohistochemical staining for keratin (wide, DAKO, Glostrup, Denmark; a dilution of 1/50), epithelial membrane antigen (EMA, DAKO; 1/30), surfactant apoprotein (SAP, DAKO; 1/100), CEA (DAKO; 1/50), desmin (DAKO; 1/50), smooth muscle actin (DAKO, 1/400), vimentin (DAKO, 1/400), Ulex europaeus I agglutinin (UEA-1; DAKO; 1/100), HMB-45 (Enzo Diagnostics, New York; 1/10), proliferating cell nuclear antigen (PCNA; Novocastra, Newcastle upon Tyne, UK, 1/25), p53 protein (mutant type, DO-7, DAKO, $1 / 40$ ), estrogen and progesterone receptors (Novacastra, USA; 1/10) was also performed with the L-SAB (DAKO), immunoperoxidase technique using a manual method. Moreover, two polyclonal antibodies for tuberins, TSC2 gene products, (one from Santa Cruz Bio, Santa Cruz, CA [1/200] and the other kindly provided by Dr. Mizuguchi, Jichi Medical University, Oyama, Japan [1/300]), were used with the Envision method (DAKO). Positive controls such as normal kidney, lung, and skin were used for each antibody. For germline mutation analysis of the TSC1 and TSC2 genes, $30-\mathrm{mL}$ blood samples from the patient and her son were taken after informed consent was obtained from them, and genomic DNA isolated from leukocytes was used for polymerase chain reaction-single-strand conformational polymorphism (PCR-SSCP) analysis according to the procedure of Orita et al. (6). When a mobility shift was detected by PCR-SSCP, direct DNA sequencing was performed. $\mathrm{LOH}$ with microdissection was performed using paraffin-embedding lung tissues.

\section{RESULTS}

\section{Histologic and Immunohistochemical Findings}

In the skin, facial angiofibroma and ungual fibroma of bilateral foots were confirmed. In the lung, multiple micronodule formations were found in the dilatation and destruction of air spaces and multiple cystlike background, the largest nodule measuring approximately $4 \mathrm{~mm}$ in diameter (Fig. 1). The center of the micronodules showed papillary or trabecular pattern. Within the micronodules, degenerated thick elastic fibers and condensed collagen fibers were seen on EVG-staining slides, to- 


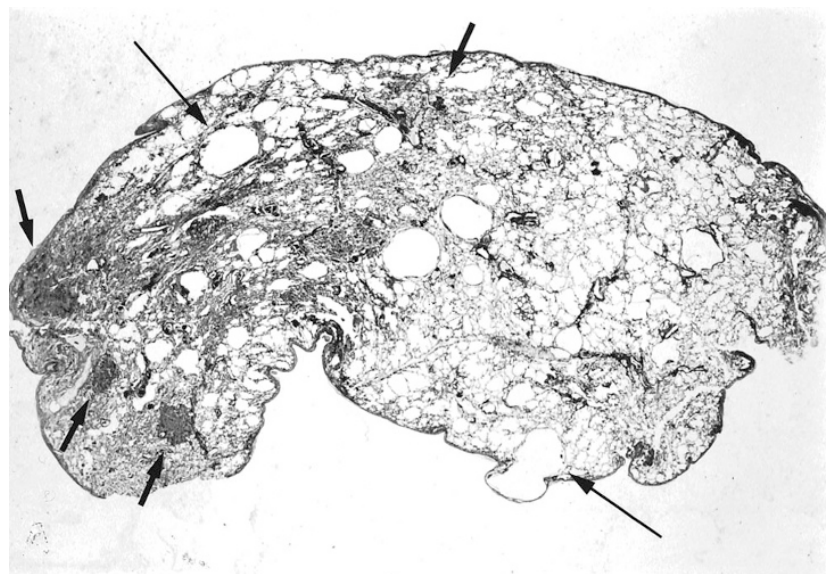

FIGURE 1. Microscopic panoramic views of the open lung biopsy from left $\mathrm{S}^{1+2}$. Multifocal micronodular pneumocyte hyperplasia (arrows) and LAM (larger arrows) are apparent (elastica van Gieson stain; original magnification, $1 \times$ ).

gether with reticulin fibers demonstrating an alveolar pattern on silver impregnation. Their edges were papillary along the surface of the alveoli without any fibrous capsule (Fig. 2). With periodic acidSchiff, the epithelial cells of the micronodules demonstrated a few intracytoplasmic eosinophilic inclusions. Mitoses were not evident. The micronodules were composed of epithelial cells with cuboidal or columnar shape and vesicular round nuclei, sometimes with clear nucleolus.

The results of immunohistochemical studies are summarized in Table 1. Immunohistochemically, keratin (Fig. 3), EMA, SAP, and CEA were positive in the cells of the micronodules, but vimentin, desmin, actin, UEA-1, and HMB-45 were negative. PCNA showed strongly positive reactivity in the cells of MMPH ( $>90 \%$ of the cells), although PCNA also reacted positively with normal pneumocytes and bronchiolar epithelia. p53 and estrogen and

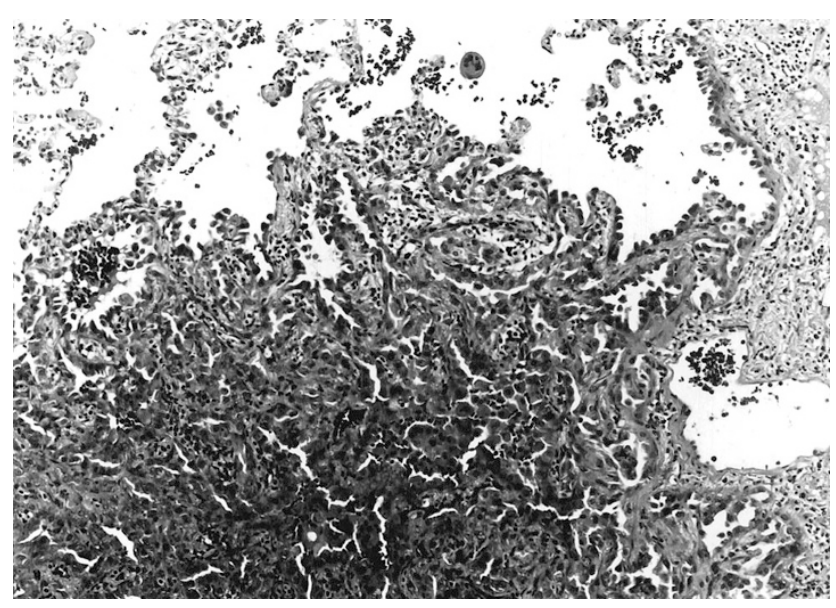

FIGURE 2. The border of the multifocal micronodular pneumocyte hyperplasia shows a papillary pattern with irregular margin and lymphocyte infiltration in the stroma (hematoxylin and eosin staining; original magnification, $100 \times$ ). progesterone receptors were negative in the cells. The micronodules were diagnosed as MMPH.

Formation of multiple small cystlike spaces was widespread from the subpleural areas to alveolar areas. In the large and small cystic walls, bundles of the spindle cells were found in leiomyomatous or fibromyomatous lesion, mainly along the alveolar ducts to bronchiolar walls. Immunohistochemically, the spindle cells were positive for desmin, smooth muscle actin, HMB-45 (Fig. 4), PCNA, vimentin (partly), and both estrogen and progesterone receptors but negative for p53. Bundles of the spindle cells were diagnosed as LAM. Moreover, tuberin was positive in LAM (Fig. 5) but negative to slightly positive for MMPH, with renal tubules and bronchial epithelia being positive as controls.

\section{Molecular Findings}

As a result, PCR-SSCP analysis of the TSC2 gene using leukocytes of the present patient detected a mobility shift on Exon 9 without any of PCR fragments amplified from the TSC1 gene. But no mobility shifts of either the TSC1 or the TSC2 gene were detected in the sample from her son. With direct DNA sequencing, superimposed sequencing ladders confirmed one nucleotide deletion of $\mathrm{G}$ (or g) between Exon 9 and the Intron 9 boundary of the TSC2 gene (a deletion of nt 975G or nt $975+1$ g; Fig. $6 \mathrm{~A}-\mathrm{C})$. With microdissection using lung tissues, $\mathrm{LOH}$ of the TSC2 gene was detected in a LAM lesion, but not MMPH, of the present patient as compared with a control (Fig. 7).

\section{DISCUSSION}

In the present paper, we document pathologic and genetic findings of a 45-year-old woman with TSC whose diagnosis has been confirmed by meeting Gomez's diagnostic criteria (2), such as facial angiofibroma, ungual fibromas, subependymal calcifications, angiomyolipomas of kidneys and liver (radiographically), and MMPH with LAM of lungs. MMPH was first described in a TSC patient without LAM by Popper et al. (4) in 1991. The MMPH (this term was introduced by Guinee et al. (7) in 1995) showed strong immunoreactivity for cytokeratin and peanut agglutinin and Ulex europaeus I aggulutin (UEA-1). It has also been in the same category as adenomatoid proliferation of alveolar epithelium (8), papillary alveolar hamartoma, multifocal alveolar hyperplasia, or multinodular pneumocyte hyperplasia (9). Since Popper's paper, eight reports covering 16 patient cases of evident MMPH in TSC have appeared in the English literature $(10,11)$. MMPH should be histologically distinguished from papillary adenoma of Type II cells, atypical adenomatous hyperplasia (AAH), bronchioloalveolar carci- 


\begin{tabular}{|c|c|c|c|c|c|c|c|c|c|c|c|c|c|c|}
\hline Cell Type & Keratin & EMA & SAP & CEA & Desmin & $\begin{array}{c}\text { Smooth Muscle } \\
\text { Actin }\end{array}$ & Vimentin & UEA-1 & HMB45 & PCNA & p53 & EgR & PgR & Tuberin \\
\hline МMPH & + & + & + & + & - & - & - & - & - & $\begin{array}{c}+ \\
>90 \%\end{array}$ & - & - & - & - \\
\hline \multirow[t]{2}{*}{$\begin{array}{l}\text { Normal } \\
\text { pneumocytes }\end{array}$} & + & + & + & + & - & - & - & - & - & + & - & - & - & - \\
\hline & & & & & & & & & & $50 \%$ & & & & \\
\hline Alveolar macrophage & - & - & $\mathrm{p}+$ & $\mathrm{p}+$ & - & - & - & - & - & - & - & - & - & - \\
\hline Endothelial cells & - & - & - & - & - & - & + & + & - & - & - & - & - & - \\
\hline Spindle cells of LAM & - & - & - & - & + & + & $\mathrm{p}+$ & - & $\mathrm{p}+$ & $\mathrm{p}+$ & - & $\mathrm{p}+$ & $\mathrm{p}+$ & + \\
\hline
\end{tabular}

MMPH, multifocal micronodular pneumocyte hyperplasia; LAM, lymphangioleiomyomatosis; EMA, epithelial membrane antigen; SAP, surfactant apoprotein; UEA-1, Ulex europaeus I agglutinin; EgR, estrogen receptor; PgR, progesterone receptor.

The reactivity was judged as positive $(+)$, partly positive $(\mathrm{p}+)$, and negative $(-)$.

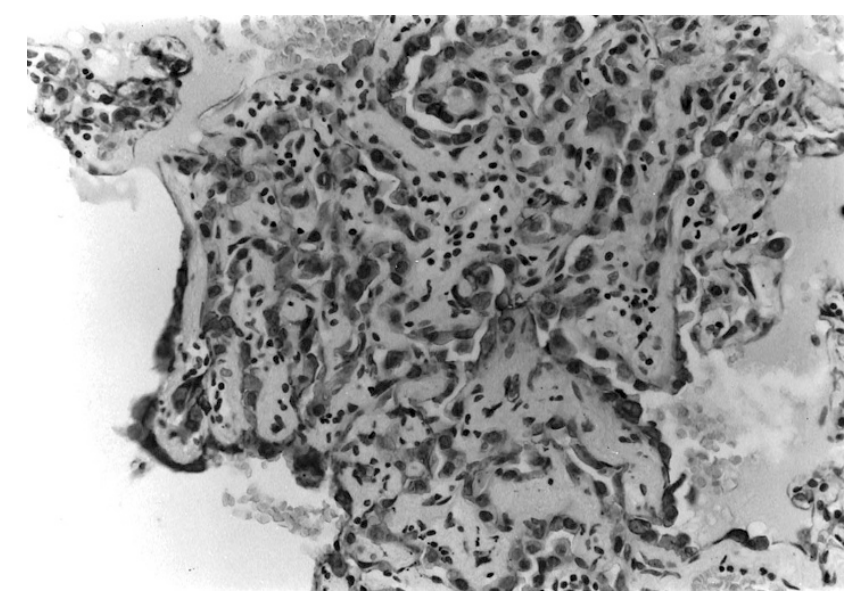

FIGURE 3. Immunohistochemically, most of the proliferating cells of multifocal micronodular pneumocyte hyperplasia are positive for keratin (wide; L-SAB method; original magnification, $200 \times$ ).

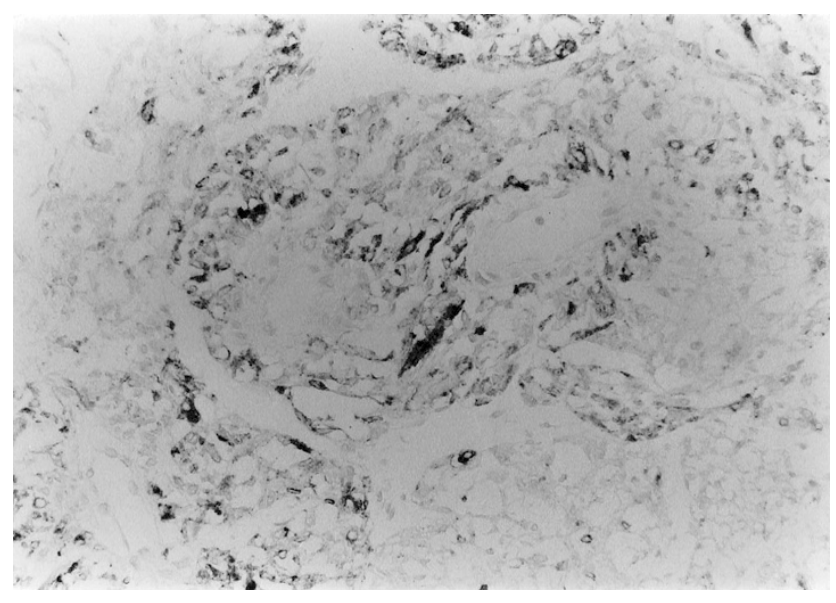

FIGURE 4. Immunohistochemically, some spindle cells of lymphangioleiomyomatosis are patchily positive for HMB-45 (L-SAB method, original magnification, 200x).

noma, and several other pulmonary benign lesions, such as sclerosing hemangioma (pneumocytoma), chemodectoma, minute pulmonary meningotheliallike nodules, and healing miliary inflammatory process (focal pneumonitis). Previous authors indicated that MMPH was distinctively a proliferative and epithelial lesion of probably hamartomatous nature that

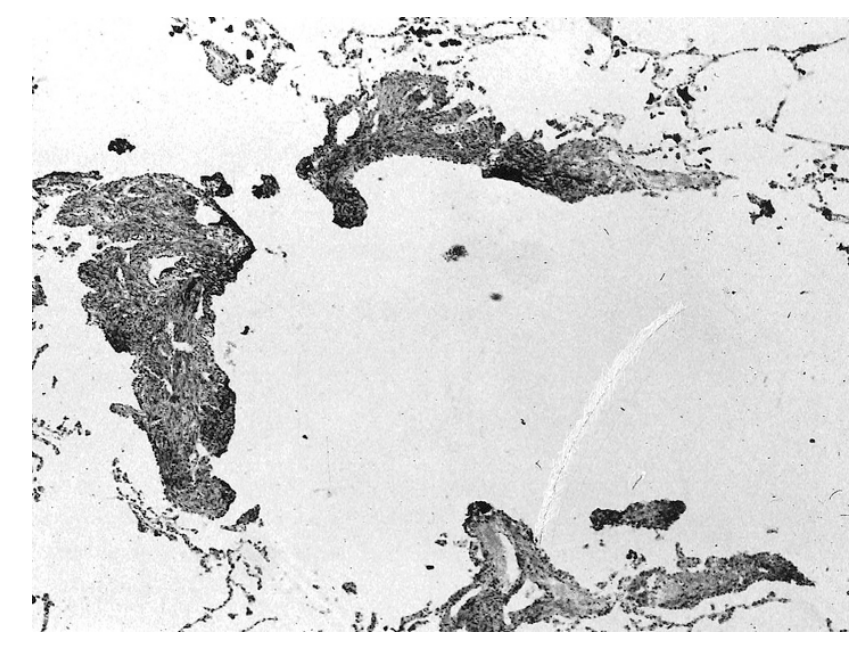

FIGURE 5. Immunohistochemically, the spindle cells of lymphangioleiomyomatosis are partially positive for tuberin (Envision method, original magnification, 40x).

appeared to be highly associated with TSC and did not possess malignant potential $(4,7-11)$. It has also been reported that a patient with pulmonary angiomyolipoma with MMPH (10) and another patient with MMPH without LAM at a postmenopausal age (11) were found among TSC patients.

The TSC2 gene is composed of $45 \mathrm{~kb}$ of genomic DNA, contains 41 coding exons, and encodes a message of $6 \mathrm{~kb}$ and a protein of 1741-1807 amino acids (12). Yamashita et al. (12) conducted the TSC2 gene analysis of Japanese patients and found six pathogenic mutations in sporadic cases but not familial cases, including two frameshifts, one frame deletion, and three missense mutations. Two of the TSC2 mutations were expected to result in a truncated tuberin gene product. Tuberin contains a region of limited homology to the catalytic domain of raplGAP (GTPase-activating protein), thought to be a member of the ras superfamily of GTPases and functioning in growth, motility, and intracellular compartmentalization. The TSC1 gene product, called hamartin, is not well known, but it might function in the same complex with tuberin (12).

It has been recently reported by Kimura et al. (5) that in a autopsy case of TSC and LAM without 


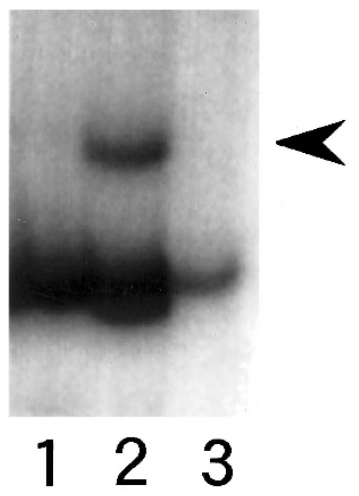

A
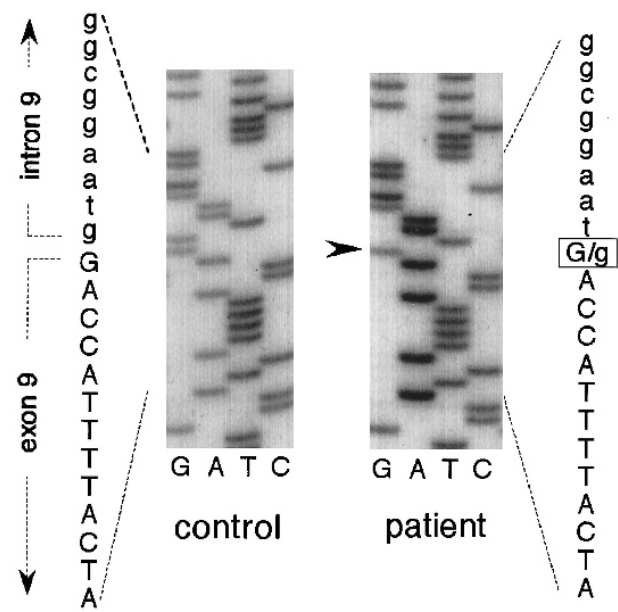

B

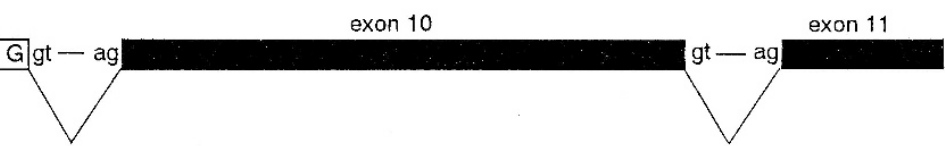

FIGURE 6. Germ line analysis of the TSC2 gene with leukocytes. A, polymerase chain reaction-single-strand conformational polymorphism analysis detected a mutation on Exon 9 of the TSC2 gene in the present patient (lane 2, arrowhead) as compared with a control (lane I) and her son (lane 3). B, direct DNA sequencing shows a deletion of G or g between Exon 9 and Intron 9 of the TSC2 gene. C, schematic representation.

MMPH, immunoexpression of HMB-45 was observed in the hamartomas of the lung and angiomyolipomas of the kidneys but that tuberin, using antituberin C20 antibody (amino acids 1765-1784), was immunohistochemically negative or only very weakly expressed but not positive. However, it has been recently reported by Muir et al. (9) that in a series of 14 patients with MMPH and/or LAM in TSC, both TSC2 mRNA (in situ hybridization) and immunoreactive positivity for tuberin was demonstrated in the Type II pneumocytes of the MMPH and the smooth muscle cells of LAM in several of the cases examined. In our study, immunohistochemical expression of tuberin (C 20 antibody and another antibody [amino acids 1770-1784] were kind gifts by Dr. Mizuguchi) was positive in LAM lesions but negative or only weakly positive in MMPH. More studies are needed to clarify the details of tuberin expression in TSC patients with TSC1 or TSC2 gene mutations.

In TSC, allelic loss or loss of heterozygosity (LOH) was first reported in 1994 for the chromosome $16 \mathrm{p} 13.3$ region where the TSC2 gene is located. $\mathrm{LOH}$ has now been reported in renal angiomyolipomas of TSC patients (12). In our present case, using a microsatellite marker (16p13.3, D16S291), preliminary results demonstrated LOH in LAM lesions (3/3) but not in MMPH (0/2). This is consistent with immunohistochemical expression in LAM, suggesting TSC2 to be a tumor suppressor gene.

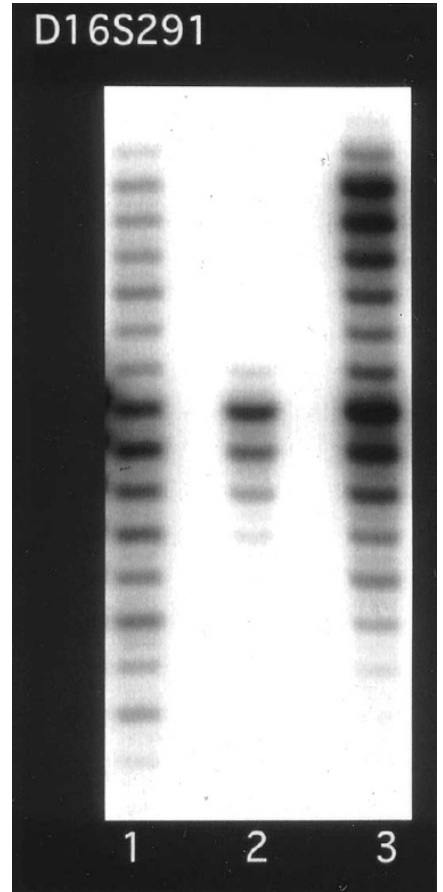

FIGURE 7. With microdissection using formalin-fixed lung tissues, loss of heterozygosity of the TSC2 gene (a marker of D16S291) was detected in a lymphangioleiomyomatosis lesion of the present patient (lane 2) as compared with a control (lane 1) and multifocal micronodular pneumocyte hyperplasia lesion (lane 3).

There is a long-standing debate whether sporadic LAM and this type of lung lesion in TSC are the same. LAM affects about $5 \%$ of women with TSC (8). 
It has been recently demonstrated by Carsillo et al. (13) that somatic mutations in the TSC 2 gene occur in the pulmonary LAM cells of women with sporadic LAM, suggesting a direct role of TSC2 gene in the pathogenesis of sporadic LAM.

In conclusion, although LAM has already been established as a pulmonary lesion of TSC, MMPH can be found as a new pulmonary lesion in TSC patients with and without LAM. Moreover, using genetic and immunohistochemical analyses, the histogenesis of hamartomatous lesions such as MMPH and LAM in TSC patients could be clarified in association with the TSC2 or TSC1 gene.

Acknowledgments: We thank Dr. Masanori Kitaichi, Associate Professor, Department of Pathology, Kyoto University Hospital, Japan, for pathologic diagnosis.

\section{REFERENCES}

1. Osborne JP, Fryer A, Webb D. Epidemiology of tuberous sclerosis. Ann N Y Acad Sci 1991;615:125-7.

2. Gomez MR. An overview: tuberous sclerosis complex. In: Niimura M, Otsuka F, Hino O, editors. Phacomatosis in Japan. Gann Monograph on Cancer Res No. 46. Tokyo: Japan Scientific Societies Press and Karger; 1999. p. 17-25.

3. Povey S, Burley MW, Attwood J, Benham F, Hunt D, Jeremiah SJ, et al. Two loci for tuberous sclerosis: one on 9q34 and one on 16p13. Ann Hum Genet 1994;58:107-27.

4. Popper HH, Juettner-Smolle FM, Pongratz MG. Micronodular hyperplasia of type II pneumocytes-a new lung lesion associated with tuberous sclerosis. Histopathology 1991;18: 347-54.

5. Kimura N, Watanabe M, Date F, Kitamoto T, Kumura I, Horii A, et al. HMB-45 and tuberin in hamartomas associated with tuberous sclerosis. Mod Pathol 1997;10:952-9.

6. Orita M, Suzuki Y, Sekiya T, Hayashi K. Rapid and sensitive detection of point mutations and DNA polymorphisms using the polymerase chain reaction. Genomics 1989;5:874-9.

7. Guinee D, Singh R, Azumi N, Singh G, Przygodzki RM, Travis W, et al. Multifocal micronodular pneumocyte hyperplasia: a distinctive pulmonary manifestation of tuberous sclerosis. Mod Pathol 1995;8:902-6.

8. Bonetti FB, Chiodera P. The lung in tuberous sclerosis. In: Corrin B, editor. Pathology of lung tumors. New York: Churchill Livingstone; 1997. p 225-39.

9. Muir TE, Leslie KO, Popper H, Kitaichi M, Gagne E, Emelin JK, et al. Micronodular pneumocyte hyperplasia. Am J Surg Pathol 1998;22:465-72.

10. Wu K, Tazelaar HD. Pulmonary angiomyolipoma and multifocal micronodular pneumocyte hyperplasia associated with tuberous sclerosis. Hum Pathol 1999;30:1266-8.

11. Yamanaka A, Kitaichi M, Fujimoto T, Hirai T, Hori H, Konishi F. Multifocal micronodular pneumocyte hyperplasia in a postmenopausal woman with tuberous sclerosis. Virchows Arch 2000;436:389-92.

12. Yamashita Y, Ono J, Okada S, Wataya-Kaneda M, Yoshikawa K, Nishizawa M, et al. Analysis of all exons of TSC1 and TSC2 genes for germline mutations in Japanese patients with tuberous sclerosis: report of 10 mutations. Am J Med Genet 2000;90:123-6.

13. Carsillo T, Astrinidis A, Henske EP. Mutations in the tuberous sclerosis complex gene TSC2 are a cause of sporadic pulmonary lymphangioleiomyomatosis. Proc Natl Acad Sci U S A 2000;97:6085-90. 